\title{
Mitteleuropäisch-jüdische Lebensgeschichte als transnationale Verflechtungsgeschichte
}

\author{
Eduard Goldstückers dialogische Erinnerungen
}

\begin{abstract}
An entanglement of several central aspects of twentieth-century European intellectual and cultural history marks the life story of the Czech literary critic Eduard Goldstücker (1913-2000). Goldstücker's life was shaped by such pivotal experiences as the Holocaust, communist rule in Eastern Europe, exile, and reform communism in Czechoslovakia. Due to these experiences and his Jewish, socialist, and transnational positions, Goldstücker's life story may be considered representative of Central European intellectual biographies. There are three different versions of Goldstücker's memoirs. The respective Czech and German texts are the product of numerous extended conversations with his former son-in-law, the Czech writer Jiři Gruša, and the German journalist, Eduard Schreiber, who served as editors for Goldstücker's memoir. The study outlines the ways in which life writing is shaped by the informant (Goldstücker), the collaborator (Gruša, Schreiber), and by the different intended audiences in the Czech Republic and Germany. It concludes that Goldstücker's life writing is the product of his Jewish as well as his socialist beliefs.
\end{abstract}

Keywords: Autobiografie, kooperatives life-writing, Verflechtungsgeschichte, Erinnerungskultur, Holocaust, Exil, Schauprozesse, Kommunismus, tschechischjüdische Kultur, Mitteleuropa

\section{Erinnerung im/als Dialog - Eduard Goldstückers Memoiren und Jiři Grušas und Eduard Schreibers Beitrag}

Wie schreibt man Lebensgeschichte zwischen Kulturen? Wer sind die Adressatinnen und Adressaten transnationaler Lebensgeschichten? Welche Form gibt man einer solchen Lebensgeschichte? Ein Blick in die lebensgeschichtlichen Texte Eduard Goldstückers (1913-2000) vermag Einblicke in die Komplexität 
transnationaler Erinnerungskulturen und ein Bewusstsein für die Schwierigkeiten lebensgeschichtlichen Erzählens über sprachliche und nationale Grenzen zu geben. Seine Biografie spiegelt in vielen Facetten die tschechoslowakische Geschichte des 20. Jahrhunderts: die Gründung der Ersten Republik, die MasarykÄra, die Shoah und das erste Exil in England während des Krieges, die antisemitischen Schauprozesse der 1950er Jahre, den Reformkommunismus der 1960er Jahre, die zweite Exilerfahrung nach 1968 und den Umbruch des Jahres 1989. Goldstücker war ein aufmerksamer Beobachter der Zeitläufte und zeitweise auch ein Akteur auf der politischen Bühne der Tschechoslowakei. Sein Selbstverständnis als mitteleuropäischer Intellektueller wurde durch die jüdische Herkunft, das frühe und nie aufgegebene Engagement für linke, sozialistische Politik und eine Liebe zur Literatur geformt. Neben seinen wissenschaftlichen Publikationen zur deutschen und Prager deutschen Literatur hat er auch lebensgeschichtliche Texte hinterlassen, die in ihrer Entstehungsgeschichte und Textform sein Leben zwischen verschiedenen Sprachen, Kulturen und Ländern spiegeln und sich aus Gesprächen heraus entwickelt haben.

Eduard Goldstückers Erinnerungen sind eminent dialogische Texte. Sie sind entstanden aus dem Gespräch der Kulturen, die ihn geprägt haben, sie sind Gegenrede $\mathrm{zu}$ herrschenden Meinungen und nicht zuletzt sind sie das Ergebnis konkreter Dialoge: Den ersten führte er mit dem Tschechen Jiří Gruša, den zweiten mit dem Deutschen Eduard Schreiber. ${ }^{1}$ Beide Male entstanden Erinnerungstexte, die man als klassische Memoiren, d.h. als eine Mischform von autobiografischer Erzählung und historischer Betrachtung, bezeichnen kann. Goldstücker interessiert sich zumeist mehr für die äußeren Ereignisse, für das „Soziale“ und lässt wenig von seiner Innerlichkeit aufscheinen. ${ }^{2}$ Auch in anderer Hinsicht folgen die Texte dem memoiristischen Erzählen: Es wird weitgehend chronologisch berichtet, und der Erzähler ist bemüht, die Erfahrungen seiner Epoche einzufangen.

1 Veronika Tuckerová, die die Akten des tschechischen Geheimdienstes zu Goldstücker im Tschechischen Nationalarchiv eingesehen hat, sieht in der den Akten beigefügten Biografie und in einem 1970 aus dem englischen Exil geschriebenen Brief weitere lebensgeschichtliche Texte. Vgl. Tuckerová $(2015,129)$. Sie liest diese Texte vor allem vor dem Hintergrund von Goldstückers Auseinandersetzung mit Kafka und vor dem Hintergrund der Bedeutung Kafkas für die autobiografische Selbststilisierung Goldstückers. Wenngleich auch diese Texte, insbesondere der Brief, dialogische Texte sind, so werden sie hier nicht mit einbezogen, da sie nicht an eine weitere Öffentlichkeit adressiert sind und als Texte, die unter Mitwirkung des StB entstanden und bewahrt worden sind, einen durchaus problematischen Status haben.

2 Er bewegt sich in dieser Hinsicht im Rahmen der Genregrenzen der Memoiren, die anders als klassische Autobiografien nicht den Anspruch erheben, Einblick in Innerlichkeit zu gewähren. Insofern greift die Kritik Müller-Funks und Budňáks nicht (Müller-Funk und Budňák 2013, 155). 
Goldstücker ist nach der Typologie Marcus Billsons: Augenzeuge, Teilnehmer und Geschichtsschreiber in einer Person (Billson 2016, 155-160). Zudem perspektivieren seine Überzeugungen den Text ideologisch. In der Ausarbeitung seiner Memoiren ist sich Goldstücker bewusst, dass seine Erinnerungen auf sehr unterschiedliche Leser und Leserinnen treffen und dass das kulturelle Imaginäre, in welches er sie hineinstellt, sehr verschieden ist und andere Narrative über das 20. Jahrhundert entwickelt hat. Die beiden dominanten Züge von Goldstückers Lebenstext - seine überzeugte, nie angezweifelte Loyalität gegenüber dem Sozialismus und dem Judentum - bringen die gleiche Haltung des intellektuellen Einspruchs zum Ausdruck, auch wenn sie sich aus unterschiedlichen Quellen speisen: Am Sozialismus hält er wider alle politischen Konjunkturen aus intellektueller Überzeugung fest, am Judentum nicht aus Religiosität, sondern aus Solidarität mit den ermordeten Opfern der Shoah und als Reaktion auf antisemitische Anfeindungen. Abhängig von Gesprächspartner und historischem Zeitpunkt der Erzählung treten diese Einstellungen in den Erinnerungstexten mehr oder weniger deutlich hervor. Die Existenz mehrerer Erinnerungstexte ist also ebenso dem Interesse seiner Gesprächspartner geschuldet wie Goldstückers Bestreben, seine Vision der tschechoslowakisch-jüdischen Geschichte im 20. Jahrhundert in die Debatte sowohl in Deutschland als auch in der Tschechoslowakei einzubringen.

Der erste Erinnerungstext entstand in Zusammenarbeit mit seinem ehemaligen Schwiegersohn, dem tschechischen Schriftsteller Jiři Gruša, noch im Exil und ist die Grundlage für die von Goldstücker bearbeitete tschechische Ausgabe, sodass bereits von zwei Versionen der Lebensgeschichte gesprochen werden muss. Der dritte Text beruht auf dem Austausch mit dem deutschen Journalisten und Dokumentarfilmer Eduard Schreiber. Der Ausgangspunkt ist in beiden Fällen der gleiche - das lebensgeschichtliche Gespräch. Die Form, in der diese Gespräche dokumentiert und in die Memoiren überführt wurden, könnte jedoch nicht verschiedener sein. Im Zuge der Verschriftlichung der Gespräche und Anordnung der Lebensgeschichte setzen die Gesprächspartner, aber auch Goldstücker unterschiedliche Akzente. Aufgrund dieser Situation liegen drei Versionen von Goldstückers Lebenserzählungen vor - zwei deutsche und eine tschechische. Die so entstandenen Lebenstexte zeichnen in Form, Inhalt und Entstehungsprozess eine für linke jüdische Intellektuelle Mittelosteuropas repräsentative Biografie nach. Zusammen gelesen zeigen die verschiedenen Versionen seiner Memoiren, dass diese Erinnerungstexte für ihn nicht nur Folge eines transnational geführten Lebens sind, sondern in seiner Wahrnehmung auch ein Modell für das Erinnern jenseits nationaler Narrative (Tippner 2019, 154-155), das Lebensgeschichte nicht ausschließlich vor dem Hintergrund einer bestimmten Kultur, sei es der deutschen, jüdischen, tschechischen oder slowakischen konzeptualisiert. Als mitteleuropäischer Jude, als Sozialist, aber auch als Komparatist und Diplomat 
denkt Goldstücker grenzübergreifend und international, wenngleich er punktuell nationale Standpunkte einbezieht und reflektiert und in seinen Erinnerungen auf nationale Dispositionen eingeht.

Mit der Arbeit an seinen Memoiren begann Goldstücker im zweiten Exil auf Anregung seines ehemaligen Schwiegersohns, Jiři Gruša. Zusammen führten sie eine Reihe langer, thematisch strukturierter Gespräche über Goldstückers Leben. Diese bilden die Grundlage der zunächst 1989 auf Deutsch unter dem Titel Prozesse: Erfahrungen eines Mitteleuropäers (Goldstücker 1989) erschienenen Memoiren. Gruša organisierte dabei nicht nur das Gespräch und stellte Fragen, er war auch für die Verschriftlichung und Gestaltung des Textes mitverantwortlich. ${ }^{3}$ Ende der 1990er Jahre arbeitete Goldstücker dann an einer tschechischen Ausgabe des von Gruša erstellten Textes, die nach Goldstückers Tod im Jahr 2000 postum in zwei Bänden unter dem Titel Vzpomínky (Erinnerungen; Goldstücker 2003; Goldstücker 2005) erschien. Während er den ersten Band dieser Ausgabe noch selbst verantwortete, wurde der zweite Band von seinen Töchtern, Anna Grušová und Helena Vávrová, herausgegeben. Beide Texte, der deutsche wie der tschechische, enden mit dem Prager Frühling und der zweiten Emigration nach Großbritannien, wenn auch auf einer anderen Note. Der erste Teil der tschechischen Ausgabe, der von Goldstücker nochmals bearbeitet wurde, wird deshalb im Folgenden als der Text betrachtet, der den Intentionen Goldstückers am nächsten kommt, der zweite Teil, der von ihm nur noch teilweise überarbeitet und postum von seinen Töchtern herausgegeben wurde, ist wiederum durch deren Wahrnehmungen mitgeformt. Die deutsche und die tschechische Fassung des auf der Basis des Gesprächs mit Gruša entstandenen Textes unterscheiden sich voneinander und entwerfen punktuell unterschiedliche Erinnerungsnarrative. Sie setzen andere Schwerpunkte, etwa wenn der tschechische Text die Shoah deutlicher hervorhebt und Manifestationen des Antisemitismus in der Tschechoslowakei herausstellt, während der deutsche Text diese spezifisch jüdischen Erfahrungen in ein größeres Narrativ einbindet. Auch im Hinblick auf die Darstellung des Kommunismus ist sich Goldstücker durchaus bewusst, dass seine deutschen Leser eine andere Einstellung zum Kommunismus und zum Prager

3 Die Bänder mit den Aufzeichnungen der Gespräche befinden sich im Gruša-Fonds des Mährischen Museums in Brno. Das Konvolut besteht aus insgesamt achtzehn Audiokassetten. Die Kassetten sind nur mit Jahreszahlen $(1987,1988)$ versehen, aber sonst nicht datiert oder beschriftet. Die Aufnahmen sind noch nicht digitalisiert und z.T. schwer verständlich. Aus dem Konvolut, das eigentlich aus achtzehn Aufnahmen besteht, konnten die Bänder 1-5 nicht gesichtet werden. Ich danke Herrn Mag. Demeter Malaták für die Möglichkeit, das Audiomaterial zu sichten. Sie werden im Folgenden zitiert als Goldstücker und Gruša (1987/1988) mit Angabe des Bandes, auf dem sich der Gesprächsausschnitt findet. 
Frühling haben als seine tschechischen Leser, die in der Transformationszeit dem Kommunismus wesentlich kritischer gegenüberstanden als das deutsche Publikum. In seiner Eigenschaft als Germanist weiß Goldstücker, dass der deutsche und tschechische kulturelle Echoraum seines Textes anders strukturiert sind.

Darüber hinaus führte Goldstücker während der Jahre 1998 bis 2000 weitere autobiografische Gespräche mit dem deutschen Journalisten Eduard Schreiber. Sie gehen über 1968 hinaus und beziehen die Jahre im zweiten Exil und die Rückkehr nach Prag 1989 in die Lebensgeschichte mit ein. ${ }^{4}$ Der in Zusammenarbeit mit Schreiber entstandene Text setzt unter dem Titel Von der Stunde der Hoffnung zur Stunde des Nichts. Gespräche (Goldstücker und Schreiber 2009) wiederum andere Akzente als der deutsche Text aus den 1980er Jahren und die tschechische Überarbeitung aus den späten 1990er und frühen 2000er Jahren. Dies liegt zum einen am historischen Abstand zu den Ereignissen, die nun auch die Wende von 1989 und die Hoffnungen, die Goldstücker in eine Erneuerung des Projekts eines Sozialismus mit menschlichem Antlitz gesetzt hatte, einschließen, und zum anderen am anders gelagerten Interesse Schreibers. Das Gespräch mit Schreiber, den der gleiche Altersunterschied von Goldstücker trennt wie Gruša, macht deutlich, dass die Unterschiede nicht so sehr den unterschiedlichen Zugangsweisen von Vor- und Nachkriegsgeneration geschuldet sind, sondern dass es hier vor allem um anders gelagerte Zugänge zur Geschichte in Deutschland und der Tschechoslowakei geht.

Wolfgang Müller-Funk und Jan Budňák sind in ihrem Aufsatz „Die zweite Spiegelung. Jiří Gruša als der Andere in Eduard Goldstückers Prozesse. Erfahrungen eines Mitteleuropäers / Vzpomínky“ der Semantik der unterschiedlichen Titel nachgegangen. Während der deutsche Text Prozesse. Erfahrungen eines Mitteleuropäers übertitelt ist und sowohl auf Kafkas gleichnamigen Roman Der Prozess wie auf die Slánský-Prozesse anspielt, aber auch Assoziationen von Entwicklung, Veränderung und Dynamik aufruft, ist die tschechische Ausgabe lapidar mit Vzpominky (Erinnerungen) betitelt und um Jahreszahlen (1913-1945 und 19451968) ergänzt. Sie sehen dies als Ausdruck eines grundsätzlichen Unterschieds zwischen beiden Texten (Müller-Funk und Budňák 2013, 148-149), der nicht zuletzt in der konkurrierenden Autorschaft begründet ist. Der aus dem Gespräch mit Schreiber entstandene Text setzt einen anderen Akzent, indem er Goldstückers Aufbrüche - aus dem slowakischen Dorf in die Großstadt Prag und vom Thoraschüler zum überzeugten Sozialisten - und die Enttäuschungen der Erwar-

4 Schreiber führte diese Gespräche als Vorarbeiten zu einem Dokumentarfilm über Goldstücker, der 2001 Premiere hatte. Die Gespräche blieben nach den Worten Schreibers „Fragment“ (Goldstücker und Schreiber 2009, 182-183). 
tungen durch Krieg, Exil und Politik betont, wobei Goldstücker gleich mehrfach vor dem Nichts stand.

Für die Auseinandersetzung mit Goldstückers Erinnerungstexten ist der kollaborative Aspekt zentral: Die Tatsache, dass an der Entstehung der Memoiren zunächst zwei, dann vier, dann fünf Personen ursächlich beteiligt sind, ist nicht nur ein Detail der Textarbeit, sondern auch ein Indiz für die Bedeutung, die das Gespräch für den Prozess des Erinnerns, seine Dynamik und die Schwerpunkte des Textes hat. Lutz Niethammer hat im Zusammenhang von oral histories darauf hingewiesen, dass latente Erinnerungen häufig erst durch „Rekonstruktionen und Assoziationen“ im Gespräch wiederauffindbar werden (Niethammer 2012, 49). Der Prozess stellt sich wie folgt dar: Es gibt eine Person (Gruša), die fragt, die die Aufnahme verantwortet und dann verschriftlicht, und eine zweite Person (Goldstücker), die ihr Leben erzählt und den verschriftlichten Text bearbeitet. Das Verhältnis zwischen Gruša und Goldstücker stellt sich in den Paratexten zur deutschen Ausgabe, die in der tschechischen Ausgabe fehlen, nicht unkompliziert dar. Jiří Grušas Erinnerungen lassen die konflikthaften Züge der Zusammenarbeit zwischen dem Dissidenten Gruša und dem Reformkommunisten Goldstücker zum Ausdruck kommen, die sich besonders an der Haltung zum Sozialismus und zur Partei entzünden. Ähnlich, doch weniger konflikthaft präsentiert sich das Gespräch zwischen Schreiber, dessen politische Auffassungen denen Goldstückers näher sind, und dem interviewten tschechischen Intellektuellen. Aber auch hier gilt, dass die Funktion des Gesprächspartners nicht nur in der Gestaltung und Aufnahme des Gesprächs besteht, sondern auch darin, einen Resonanzraum für die autobiografische Narration und auch die bereits kursierenden biografischen Interpretationen $\mathrm{zu}$ schaffen. Das Gespräch mit Schreiber enthält weniger persönlichen und historischen Zündstoff und verläuft sachlicher.

Jiři Gruša selbst hat ebenfalls ein lebensgeschichtliches Interview mit dem tschechischen Literaturwissenschaftler Dalibor Dobiáš geführt, das im Jahr 2004 unter dem Titel Umění stárnout (Die Kunst alt zu werden) im Paseka-Verlag veröffentlicht wurde. Diese Lebenserzählung präsentiert sich - anders als Goldstückers Memoiren - als Gespräch: Die Fragen des Interviewers sind ein Teil des Textes und sie tragen deutlich zur Entwicklung der Narration bei, auch wenn Gruša Themen und Motive bestimmt. Die Erzählung schreitet mehr oder weniger chronologisch voran, wobei ein deutlicher Schwerpunkt auf den 1960er bis 1980er Jahren liegt. Der Interviewer und Herausgeber Dalibor Dobiáš ergänzt die Aussagen Grušas punktuell um historische Dokumente wie etwa die Erinnerungen anderer Zeitzeugen, aber auch Texte Grušas selbst zu den angesprochenen Themen. Im Gespräch mit Dobiáš macht Gruša deutlich, dass die Initiative zur Niederschrift der Erinnerungen Goldstückers Mitte der 1980er Jahre von 
ihm ausging. Er begründet dies damit, dass Goldstücker ungern und langsam geschrieben habe, sodass er seinem ehemaligen Schwiegervater vorgeschlagen habe, Gespräche zu führen, die er dann verschriftlichen würde.

Gruša und Goldstücker handeln in den einzelnen Gesprächssitzungen bestimmte Themen ab: die Haft in Leopoldov, die Zeit als Diplomat in Israel, Goldstückers Arbeit an der Karls-Universität. Die Gespräche werden zu großen Teilen von Goldstücker bestritten, der aus dem Gedächtnis berichtet. Wenn er abschweift, versucht Gruša, ihn wieder in die thematischen Bahnen der Sitzung zu lenken. Stellenweise bittet er um Präzisierung, was Daten und Dauer von bestimmten Ereignissen betrifft. Punktuell fragt er nach und lässt sich etwas erklären, kommentierend oder wertend greift er kaum ein. Kommentare oder Interpretationen werden von Goldstücker nicht selten deutlich zurückgewiesen, etwa wenn er zu Gruša sagt: „Nein, das ist von Dir konstruiert.“5 An anderen Stellen wird deutlich, dass Goldstücker sich vorbereitet hat und seine Erinnerungen bereits ein wenig geordnet hat, etwa wenn er sagt: „Ich fange damit an, dass ....“ oder ,jetzt überspringe ich etwas ...“ oder längere Ausführungen über Israel als „Referat“ bezeichnet. ${ }^{6}$ Alles in allem ist der Umgang vertraut und der Ton freundlich, nicht selten wird gemeinsam gelacht, über die Anekdoten oder Witze, die Goldstücker erzählt. Bestimmte Anekdoten, Wendungen und Einschätzungen tauchen mehrfach in den Erzählungen Goldstückers auf und sind ein Indiz dafür, dass er manche Geschichten schon häufiger erinnert und erzählt hat. ${ }^{7}$ Aus dem Audiomaterial erstellte Gruša dann einen schriftlichen Text, der die Mündlichkeit weitgehend ausblendet, indem er seine Fragen und viele der Anekdoten nicht aufnimmt. Bereits die Gespräche enthalten wenig Privates Goldstücker gibt kaum über Gefühle Auskunft, sondern konzentriert sich auf die äußeren Ereignisse, der verschriftlichte Text beschränkt sich dann mit wenigen Ausnahmen auf das öffentliche Leben und klammert Privates aus.

Gruša beschreibt den Arbeitsprozess wie folgt: „Er hat jedes Kapitel autorisiert, wir haben es übersetzen lassen. Goldstücker bestand nämlich auf Tschechisch. Auch der Verleger schickte Kapitel für Kapitel an den Erzähler. Das Buch war schon in den Druckfahnen, als man mich anrief, dass Goldstücker sich verzerrt dargestellt fühle. Ich war völlig baff. “8 Goldstücker lässt sich die Deutungs-

5 „Ne, to je tvoje konstrukce.“ Goldstücker und Gruša (1987/1988, Band 9). Diese Äußerung fällt im Zusammenhang von Goldstückers Erinnerungen an Israel und Grušas Nachhaken in Bezug auf seine jüdische Identität.

6 Vgl. Goldstücker und Gruša (1987/1988, Band 17).

7 Dies wird auch daran deutlich, dass manche Geschichten in sehr ähnlicher Form in dem Interview Eduard Schreibers auftauchen.

8 „Každou kapitolu schvaloval, dávali jsme to překládat. Goldstücker totiž trval na češtině. Také 
hoheit über sein Leben, aber auch die Beurteilung der historischen Ereignisse nicht nehmen und hat den Prozess der Verschriftlichung als eher mechanisch aufgefasst. Im Vorwort zur deutschen Ausgabe definiert er Jiři Grušas Funktion als die eines Schreibers oder Ghostwriters und dankt ihm „für die Bereitschaft mein Gesprochenes in sein Geschriebenes umzuwandeln“ und er weist darauf hin, dass Gruša ihm „einen großen Gefallen getan [habe]“ und dass auch „nach meiner notwendigen Überarbeitung [...] im Text eine Spur einer äußeren Spiegelung übriggeblieben [sei], jener in seinen Augen“ (Goldstücker 1989, 9). Auffällig ist, dass die tschechische Ausgabe die Anmerkungen zur Genese vollkommen unterschlägt und damit die Dialogizität noch weiter zurücknimmt. In Grušas Erinnerung aus dem Interview-Band Umění stárnout (Kunst alt zu werden) erklärt sich Gruša Goldstückers Rückzieher wie folgt: „Dann schoss es mir aber durch den Kopf: Er kehrt in die Partei zurück. Das kommende Jahr 1989 erschien den ehemaligen Reformkommunisten als eine Reprise von 68. Die Wiedergeburt der Wiedergeburt [ein neuer Prager Frühling].“9

Gerade im Kontrast mit Eduard Schreibers Version von Goldstückers gesprochenen Memoiren tritt Grušas Verfahren deutlicher hervor. Der kulturelle und politische Kontext hatte sich in den zehn Jahren, die zwischen den Gesprächen liegen, verändert. Goldstücker lebte nicht mehr im Exil und seine Hoffnungen auf einen sozialistischen Neuanfang haben sich nicht erfüllt. Schreiber ist als Gesprächspartner wesentlich weniger konfrontativ, auch wenn er durchaus eigene Schwerpunkte verfolgt. Während in dem von Gruša verantworteten Text Erinnerungen (Goldstücker), historische Perspektive und literarische Imagination (Gruša) verschmelzen, ${ }^{10}$ bleiben sie bei Schreiber erkennbar getrennt. Schreibers Versuche, beispielsweise Erfahrungen Goldstückers zu generalisieren und ihnen eine kollektive Bedeutung, insbesondere für die jüdische Gemeinschaft in der Tschechoslowakei zuzuschreiben, wird von Goldstücker zwar punktuell akzeptiert, in der Regel aber zurückgewiesen. Ähnlich wie er Grušas Versuche, sein Wirken in Israel im Licht einer zionistischen oder zumindest jüdischen Loyalität zu lesen, im Gespräch scharf negierte. ${ }^{11}$

Für Eduard Schreiber ist Goldstücker vor allem der Zeitzeuge der untergegangenen mittelosteuropäisch-jüdischen Welt, als deren Repräsentant sich

\footnotetext{
nakladatel posílal kapitolu po kapitole narátorovi. Kniha už byla ve stránkách, když mi telefonovali, že se Goldstücker cítí být zkreslen! Byl jsem úplně paf.“ (Gruša und Dobiáš 2004, 124) 9 „Pak mi ale blesklo: vrací se do strany. Nastávající rok 1989 se někdejším reformistům jevil jako repríza rok '68. Obrodák obrodáku.“ (Gruša und Dobiáš 2004, 124)

$10 \mathrm{Zu}$ dieser Unterscheidung vgl. Stone (1982, 151-165).

11 Vgl. Goldstücker und Gruša (1987/1988, Band 9).
} 
Goldstücker selbst wahrnimmt, auf die er sich jedoch nicht reduziert sehen will. Wenn Schreiber etwa die These aufstellt, dass „die wesentlichen Dinge in Prag [...] aus dem Jüdischen kamen“ (Goldstücker und Schreiber 2009, 44), dann geht Goldstücker lediglich en passant darauf ein, indem er die Meinung anderer zitiert. Schreibers Ansinnen, dass sein glückhaftes Überleben Folge eines ,jüdischen Gens“ sei (Goldstücker und Schreiber 2009, 127-128), wehrt Goldstücker mit den Worten ab, dies sei „nur in dem Sinne Glück, dass [ich] all die Gefahren, die meinem Leben drohten, überlebt habe“. Während der von Gruša verantwortete Text die Singularität der Lebensgeschichte ausstellt und kaum Ansprüche auf eine Transzendenz des Individuellen, beispielsweise als Generationserfahrung erhebt, bringen Schreibers Fragen das Bemühen zum Ausdruck, Goldstücker als Vertreter einer untergegangenen Kultur zu präsentieren. Dies macht sich im Deutschen auch sprachlich bemerkbar: Goldstückers Sprachstil wird in den von Schreiber herausgegebenen Gesprächen im Unterschied zu den von Friedrich Uttitz übersetzten Erinnerungen, an ein (imaginäres) Prager Deutsch angelehnt. Darüber hinaus versucht Schreiber die Mündlichkeit zumindest teilweise zu erhalten (Schreiber 2009, 183).

Es sind jedoch nicht nur diese konträren politischen Einstellungen und der divergierende Blick auf einzelne Lebensphasen und nicht zuletzt die Bedeutung, die der tschechischen Kultur zugeschrieben wird (Gruša denkt stärker europäisch, Goldstücker national), die schließlich zum Zerwürfnis zwischen Gruša und Goldstücker führen, sondern das Spannungsverhältnis, das in die Zusammenarbeit eingeschrieben ist. Gruša, der eigentlich der bekanntere Autor ist, fungiert hier als Ghostwriter, der, wenn schon nicht anonym, so doch im Hintergrund agiert; Goldstücker, der das Leben geführt hat, das Romanautoren inspirieren kann (wie er auf einem der Bänder sagt (Goldstücker und Gruša 1987/1988, Band 17)), vermag selbst nicht seinen Lebensroman aufzuschreiben. Am Ende ist Grušas Gestaltungswille mindestens ebenso groß wie Goldstückers Anspruch auf die Deutungshoheit über sein Leben. Ein Widerspruch, der nicht $\mathrm{zu}$ überbrücken ist.

Vor dem Hintergrund der vielen historischen Ereignisse, die der Diplomat und Literaturprofessor als Zeitzeuge miterlebte, fällt die prominente Stellung auf, die in allen drei Texten seinem lebenslänglichen politischen Engagement für den Sozialismus, seiner Erfahrung als tschechoslowakischer Jude und seiner Arbeit als Literaturwissenschaftler zugewiesen wird. Antisemitismus und Antizionismus, die Shoah, aber auch eine transnationale kulturelle Prägung - diese spezifisch jüdischen Erfahrungen - bilden, neben der lebenslangen sozialistischen Orientierung, den basso continuo von Goldstückers Leben und werden durch die Leitmotive der Diskriminierung und die stetige Erinnerung an die Shoah an zentralen Stellen in allen drei Lebenstexten häufig aufgerufen. 


\section{Unverbrüchliche Loyalitäten I - Jüdische Identität als „Schicksal“}

Fragen jüdischer Identität bilden den Ausgangs- wie den Endpunkt seiner mit Gruša erarbeiteten Memoiren. Goldstücker lässt keinen Zweifel daran, dass sein Leben im Schatten der Shoah stand. Dem ersten Band seiner Erinnerungen stellt er in der tschechischen Fassung einen Prolog voran. Hier heißt es (anders als in der deutschen Fassung):

Im Exil in Brighton habe ich mir am 10. Juli 1974 aus heiterem Himmel notiert: Am heutigen Tag, dem 10. Juli 1974, wäre mein einziger Neffe Josef Zelmanovič vierzig Jahre alt geworden... Als er zehn Jahre alt war, wurde er gemeinsam mit seiner Mutter und der Großmutter, meiner Schwester Jolana und meiner Mutter Terezia von Kaschau nach Auschwitz deportiert und ins Gas geschickt. [...] Ich allein habe überlebt und gedenke meiner Toten immer an ihrem Geburtstag und des einzigen, dessen Todestag ich kenne, meines Vaters, auch an seinem Todestag. (Goldstücker 1989, 11) ${ }^{12}$

Im nächsten Absatz des Prologs fragt sich Goldstücker, ob die Erinnerung an den Neffen für seine Überlegungen zur Shoah und zum Totengedenken nicht eine Art Rückkehr des Unterdrückten bedeuteten und ein Hinweis darauf seien, wie sehr ihn sein „mitteleuropäisch-jüdisches Schicksal belaste“ (Goldstücker 2003, 5; Goldstücker 1989, 12). Die deutsche Fassung integriert diese Überlegungen in das erste Kapitel und trennt die Überlegungen zu einem spezifisch mitteleuropäischjüdischen Schicksal vom Kaddisch für den toten Neffen und die anderen ermordeten Verwandten durch einen längeren Abschnitt über Goldstückers Vater und seine Schwester, die einzigen Familienmitglieder Goldstückers, die eines natürlichen Todes gestorben sind. Das Vorwort, das im tschechischen Text die Schwelle zur Erinnerung bildet, um mit Gérard Genette zu sprechen (Genette 1989, 10), ist auch eine Vorschrift (Wirth 2004, 607). Da, wo der tschechische Text eine Leseanweisung gibt und Judentum und Shoah so als Voraussetzung von Erinnerung und Leben darstellt, sind sie im deutschen Text in die Lebensgeschichte eingeordnet und anderen formativen Erfahrungen gleichgestellt. Auch die deutsche Ausgabe verfügt über ein Vorwort, es ist jedoch anders gelagert, und wendet sich weniger der

12 „V brightonském exilu jsem si 10. července 1974 zničehonic poznamenal: Dnes by bylo mému jedinému synovci, Jozefu Zelmanovičovi, čtyřicet let ... Když mu bylo deset, odtransportovali ho někdy v červenci s matkou a babičkou, s mou sestrou Jolanou a mou matkou Tereziî z Košic do Osvětimi a poslali do plynu. [...] Já jediný jsem zůstal naživu a vzpomínám na své mrtvé vždy o jejích narozeninách a $u$ jediného $z$ nich, jehož datum smrti opravdu znám, u svého otce, $v$ den jeho smrti.“ (Goldstücker 2003, 5) 
Vergangenheit als der Gegenwart des Schreibens zu. Zwar wacht auch hier ein totes Kind - Goldstückers Enkel Martin Gruša ${ }^{13}$ - an der Schwelle, aber die Rahmung durch die Shoah ist wesentlich weniger markiert. Hier bezieht sich die Vorschrift auf die Textgenese und betont die Entstehungssituation des Textes im Exil.

Die unterschiedliche Akzentuierung von Shoah, Antisemitismus und Sozialismus hat einerseits mit seinen Gesprächspartnern zu tun, die unterschiedliche Fragen stellen, andererseits aber auch damit, dass Goldstücker sein jeweiliges Publikum, also deutsche oder tschechische Leser und Leserinnen, im Blick hatte. So erklärt er den deutschen Lesern im Kapitel über Kafka, in dem er noch einmal auf die tschechisch-deutschen Beziehungen im Schatten der Shoah eingeht, dass er nicht an eine Kollektivschuld glaube (Goldstücker 1989, 286). Er erläutert dies mit seiner slowakisch-jüdischen Herkunft, die ihn im tschechisch-deutschen Geschichtstheater zum Zuschauer, aber nicht zum Beteiligten gemacht habe:

Da ich aus der Slowakei nach Böhmen gekommen war, aus einer jüdischen Familie stammte
und mich bei den Tschechen erst eingelebt hatte, ließ mich die Erblast unberührt [...]. Die
deutsch-tschechischen Beziehungen konnte ich daher wie von außen betrachten, objekti-
ver als ein gebürtiger Tscheche oder Deutscher, ohne Affekt. Niemals, auch nicht nach dem
Krieg, identifizierte ich Deutsche pauschal mit Nationalsozialisten. (Goldstücker 1989, 285)

Der tschechische Text platziert diesen Passus an anderer Stelle, aber gleichfalls im Kontext von Goldstückers germanistischer Beschäftigung mit der deutschen Literatur. Im tschechischen Text wird der letzte Satz allerdings noch um einen Einschub erweitert, der noch einmal explizit auf das Schicksal seiner Familie Bezug nimmt. Hier heißt es: „Niemals, auch nicht vor dem Hintergrund der tragischen Erfahrung meiner Familie, habe ich Nationalsozialisten und deutsches Volk gleichgesetzt. “14 Bemerkenswert ist an dieser Stelle vor allem die Tatsache, dass Goldstücker diese Überlegungen zum Umgang mit den Deutschen nach dem Krieg nicht nur in den Kontext der Shoah stellt, sondern auch breitere nicht-jüdische tschechische Haltungen miteinbezieht. Es sind solche kleinen Verschiebungen, Auslassungen und Ergänzungen, in denen sich die unterschiedliche Adressierung der Texte manifestiert.

Die Erinnerung an die Shoah eröffnet in der tschechischen Ausgabe auch den zweiten Band. Das erste Kapitel des zweiten Bandes, das den Titel „Konec války“

13 Auch sein Tod steht im Kontext totalitärer Politik, wie Gruša in seinen Erinnerungen andeutet, wenn er einen Zusammenhang zwischen der schlechten medizinischen Versorgung des Sohnes und dissidentischen Aktivitäten herstellt (Gruša und Dobiáš 2004, 236-237).

14 „Nikdy, ani na základě tragických rodinných zkušeností, jsem nekladl rovnítko mezi nacisty a německý národ.“ (Goldstücker 2005, 116 - kursiv A.T.) 
(Kriegsende) trägt, beginnt mit einem Hinweis auf die „Endlösung“, auf Konzentrationslager und auf damals noch nur verschollen geglaubte Familienangehörige (Goldstücker 2005, 7). Diese Überlegungen fehlen im deutschen Text zum Teil, zum Teil sind sie in ein längeres Kapitel mit der Überschrift „Krieg“ integriert (Goldstücker 1989, 147). Im deutschen Text wird die Shoah dem Krieg zugeordnet, im tschechischen Text ist das Wissen um sie Teil der Nachkriegszeit und Nachkriegserfahrung. Ähnlich verhält es sich mit dem Epilog des Textes, auch hier weichen deutscher und tschechischer Text wieder signifikant voneinander ab. Zwar enthalten beide Versionen, die deutsche wie die tschechische, eine Auseinandersetzung mit dem Antisemitismus, der Goldstücker in der Endphase des Prager Frühlings entgegenschlug und der sich in anonymen Briefen, aber auch in offiziellen Stellungnahmen Luft machte (Goldstücker 1989, 326; Goldstücker 2005, 154). Für Goldstücker lassen die antisemitischen Anfeindungen die Erinnerung an die Zeit der Slánský-Prozesse wieder wach werden. ${ }^{15}$ Während er sich und seinen deutschen Lesern am Ende die Frage stellt, wie der Glaube an die Utopie des Kommunismus zu bewerten sei und ob es in den Augen der Nachgeborenen mildernde Umstände gäbe, zitiert er im tschechischen Text aus der Anklageschrift des Jahres 1951. Der mit den Worten „Krátce z dokumentư“ (Kurz aus den Dokumenten) überschriebene Epilog schließt den Kreis zu den Schicksalsjahren 1938 und 1951. Die von Goldstücker zitierten Passagen aus dem Jahr 1951 haben nicht nur eine frappante Ähnlichkeit zu den Vorwürfen, die ihm nach 1968 gemacht werden und in denen erneut von ,jüdischer Verschwörung“, von „Spionage“ und zionistischen Umtrieben die Rede ist, sondern auch zur nationalsozialistischen Propaganda. Eine Verbindung, die der deutsche Text nicht herstellen kann oder will.

Auch die Gespräche mit Eduard Schreiber legen einen klaren Schwerpunkt auf Goldstückers Erfahrungen als Jude. Dies ist nicht zuletzt durch Schreibers Frageinteresse begründet, in dem sich eine deutsche Perspektive auf Mittelosteuropa manifestiert, die die Shoah als das Epochenereignis des 20. Jahrhundert versteht, während in Goldstückers Perspektive eine Gleichrangigkeit von Nationalsozialismus und Stalinismus im Hinblick auf ihre Bedeutung für die tschecho-slowakische Geschichte besteht. Goldstücker weist mehrfach darauf hin, dass seine jüdische Identität vor allem auch in der negativen Adressierung und

15 Dass er die Verbreitung des Antisemitismus nicht nur auf die 1960er Jahre beschränkt sieht, macht eine Anmerkung deutlich, die sich allerdings nur im deutschen Text findet. Hier weist Goldstücker darauf hin, dass in einem Text noch 1986 antisemitische Anschuldigungen gegen ihn erhoben wurden (Goldstücker 1989, 327). 
Behandlung durch andere gründet und dass er sich als „Mitglied einer ungeliebten Minderheit““ (Goldstücker und Schreiber 2009, 151) betrachtet.

Belastet mich mein mitteleuropäisch-jüdisches Schicksal wirklich so sehr? Für einen Großteil meines Lebens dachte ich, es wäre mir gelungen, mich aus seinen Fängen zu befreien. (Goldstücker 1989, 12)

Der von Müller-Funk und Budňák formulierte Vorwurf, dass Goldstücker „,seine jüdische Herkunft im Sinne einer klassischen linken und kommunistischen Identität hintanhält“ (Müller-Funk und Budňák 2013, 145), lässt sich vor dem Hintergrund der oben angeführten Rahmung durch Shoah und Antisemitismus nicht nachvollziehen. Die von Müller-Funk und Budňák an Goldstücker gerichtete Kritik „nicht jüdisch genug“ zu sein, wird häufig an jüdische Autoren und Autorinnen herangetragen. Sander Gilman setzt sich damit in seinem Aufsatz „Gibt es neue ,Ostjuden“ in der deutsch-jüdischen Gegenwartsliteratur?“ anhand literarischer Beispiele u.a. von Vladimir Vertlib und Wladimir Kaminer auseinander. Er interpretiert diesen Vorwurf als Ausdruck einer deutschen Erwartungshaltung und philosemitischer Idealvorstellungen (Gilman 2011, 260).

Beide Teile der Erinnerungen sind von einer durchgängigen Auseinandersetzung mit jüdischen Lebenswelten geprägt, wenngleich sie im ersten Teil den größeren Raum einnimmt. Goldstücker erinnert das jüdische Leben auf dem Dorf in der Slowakei, jüdischen Handel, die Synagoge, den Besuch des Gymnasiums und erste bittere Erfahrungen mit slowakischem Nationalismus und Antisemitismus. Vor dem Hintergrund der weitgehend dem Zeitstrahl folgenden Erzählung kommt den in den Text eingestreuten Pro- und Analepsen eine wichtige Kommentarfunktion zu. Dieses fore- und backshadowing richtet sich fast immer auf Erfahrungen jüdischer Diskriminierung und antisemitischer Angriffe, denen er sich ausgesetzt sieht und die er so zu einer kontinuierlichen Lebenserfahrung verbindet. Die Anachronien finden sich im geschriebenen Text häufiger als in der gesprochenen Version, in der Goldstücker meist versucht nicht über den Wissenstand zum Zeitpunkt des Erlebens hinauszugehen. So verbindet er die Beschreibung antisemitischer Diskriminierung im Gymnasium in Kaschau mit einer Anekdote aus einem Verhör des Jahres 1953 (Goldstücker 2003, 42), an anderer Stelle verbindet er die Erinnerung an die Hetzpropaganda tschechischer Nationalisten mit antisemitischen Verleumdungen des Jahres 1968 (Goldstücker 2003, 110-111) und macht auf diese Weise deutlich, dass der Antisemitismus vor der Shoah vieles gemeinsam hat mit dem Antisemitismus im Sozialismus.

Gerade in Bezug auf die Zeit vor seiner Verhaftung sieht er in der Rückschau die Anzeichen für einen politischen Wandel, die er damals nicht zu deuten wusste, und beschreibt seine Überlegungen als Einsichten „ex-post“ in die kom- 
menden, auch antisemitisch motivierten Repressionen (Goldstücker und Gruša 1987/1988, Band 9). So erinnert er sich an die Aufforderung, seinen jüdischen Namen zugunsten eines tschechischen aufzugeben (Goldstücker 2005, 18). Ein Ansinnen, das er mit den Worten kommentiert, dies sei der Name, mit dem seine Mutter in den Tod gegangen sei und von dem er sich niemals trennen könne. Im Gespräch mit Schreiber geht er ebenfalls auf diese Episode ein und beschreibt seinen Namen als „Schibboleth“, als Erkennungszeichen und Verstörung zugleich (Goldstücker und Schreiber 2009, 150). In Goldstückers Selbstbeschreibung als „mitteleuropäischer Intellektueller“ (Goldstücker 1989, 12, 343) ist beides aufgehoben, seine jüdische und seine politische Sozialisation vor dem Hintergrund der totalitären Erfahrung des 20. Jahrhunderts. Die jüdische Herkunft, so wird deutlich, ist für ihn stetig von Bedeutung, ohne dass er sich als Mitglied einer jüdischen Gemeinde versteht oder zionistische Neigungen hegt. Es sind, wie die angeführten Beispiele zeigen, nicht zuletzt die antisemitischen Adressierungen, die Goldstücker sein Judentum nie vergessen lassen.

\section{Unverbrüchliche Loyalitäten II - Kommunismus als intellektuelle Heimat}

In den Gesprächen mit Dalibor Dobiáš wird deutlich, dass zwischen Gruša und Goldstücker eine grundsätzliche Differenz in der Bewertung des Marxismus bestand. Während sich Gruša recht früh eine kritische Einstellung zu eigen machte, hielt Goldstücker an seinen marxistischen Überzeugungen fest. Seine Loyalität der Partei gegenüber ist angesichts seiner traumatischen Erfahrungen während der Slánský-Prozesse und der folgenden Lagerhaft sowie der antisemitischen Anfeindungen, denen er nochmals um 1968 ausgesetzt war, bemerkenswert. Gruša reagiert auf Goldstückers Bekenntnis zum Marxismus im Angesicht von Antisemitismus und Stalinismus mit Unverständnis, worauf Goldstücker ihm erklärt, dass seine Bindung an die Partei rein rational nicht zu verstehen sei und dass es eine emotionale Komponente gäbe, die er nicht unterschlagen könne, und erklärt, je stärker der Glaube, desto schwächer das Kritikvermögen (Goldstücker 1989, 81; Goldstücker 2003, 86). In dem Gespräch mit Schreiber verwendet er diesen Begriff wieder, um sein Festhalten am Marxismus zu begründen (Goldstücker und Schreiber 2009, 95, 143). Nanci Adler weist darauf hin, dass das Konzept „Glaube“ in den Narrativen der überzeugten und loyalen Kommunisten eine zentrale Rolle spielt. Sie macht darauf aufmerksam, dass es, wie bei Goldstücker, oft zu einer Spaltung kommt: Der Glaube an die Parteiführung geht verloren, der Glaube an die Überlegenheit der sozialistischen Ideologie und Weltordnung bleibt bestehen (Adler 2010, 214). 
An dieser Stelle verschärft sich im Gespräch der sonst so freundliche Ton, und das Gespräch zwischen Gruša und Goldstücker bricht ab. Auch die offensichtlichen Defizite des sozialistischen Systems in der Tschechoslowakei etwa in puncto Redefreiheit und Zensur oder Lebensstandard nimmt Goldstücker nach Grušas Ansicht nicht ausreichend zur Kenntnis. Gruša, der sich in den 1960ern als Autor, vor allem aber auch als Herausgeber der ersten nicht-dezidiert sozialistischen oder politischen Literaturzeitschrift - Tvár̆ (Gesicht) - einen Namen gemacht hatte, versteht sich selbst als „antitotalitärer“ und anti-ideologischer Schriftsteller und steht damit in einem deutlichen Gegensatz zu seinem politisch engagierten ehemaligen Schwiegervater. In der Darstellung der ideologischen Auseinandersetzungen der 1960er und 1970er Jahre stellt Gruša stets Fragen der Integrität, Loyalität und Literarizität über politische Haltungen, wobei sein politisches Ziel durchaus der Fall des sozialistischen Regimes in der Tschechoslowakei ist. Er war im Exil intensiv um die Herstellung einer Gegenöffentlichkeit und Gegenerinnerung zu den Narrativen der Normalisierungszeit bemüht. Goldstückers Kritik und Änderungswünsche am Text stellen sich für ihn als Folge dieser divergierenden Bewertung des Sozialismus dar. Gruša wie auch die Literaturwissenschaftler Wolfgang Müller-Funk und Jan Budňák bringen oft unausgesprochen, manchmal aber auch explizit, die Überzeugung zum Ausdruck, Goldstücker hätte seine kommunistischen Überzeugungen erklären, wenn schon nicht entschuldigen müssen. Insbesondere sein Festhalten am Marxismus nach den Erfahrungen der Slánský-Prozesse und nach der Niederschlagung des Prager Frühlings durch die Truppen des Warschauer Paktes rufen Unverständnis und Abwehr hervor. So etwa auch bei Veronika Tuckerová, die sich in ihrer Lesart gegen eine „westliche“ Wahrnehmung Goldstückers als heroischem Regimegegner wendet und in seinem Umgang mit der eigenen kommunistischen Vergangenheit vor allem Strategien des Verschweigens und der Selbsttäuschung entdeckt (Tuckerová 2015, 130). ${ }^{16}$ Goldstücker ist jedoch nicht defensiv, nicht einmal ambivalent, wenn es um Ziele und Realisierung marxistischer Ideale geht. An dieser Haltung hat sich auch in den späten 1990er Jahren, zum Zeitpunkt der Gespräche mit Eduard Schreiber, nichts geändert. Im Gegenteil, Goldstücker sieht in der Transformation nach 1989 eine verpasste Chance, die Reformideen eines Sozialismus mit menschlichem Antlitz doch noch zu realisieren und äußert sich sehr kritisch über Havel, Klaus und die neue liberale Politik (Goldstücker und Schreiber

16 Im Gegensatz beispielsweise zu Ingrid Hudabiunigg (2010, 282-283) betrachtet sie die Haft in den 1950er Jahren nicht als Wendepunkt. Nach Tuckerovás Interpretation weicht Goldstücker in seinen Texten der entscheidenden Frage nach seiner Mitschuld (sowohl durch sein erzwungenes Geständnis als auch durch sein Festhalten an der Partei) aus (Tuckerová 2015, 159-160). 
2009, 145). ${ }^{17}$ In seiner Wahrnehmung agiert „die neue Equipe“ wieder „dogmatisch“ und geht hinter Positionen zurück, die er bereits im Reformkommunismus der 1960er Jahre erreicht sieht. Seine Ablehnung der politischen Situation in Prag in den 1990er Jahren geht soweit, dass er davon spricht, in Prag in der ,inneren Emigration“ zu sein (Goldstücker und Schreiber 2009, 159).

Goldstückers Beharren auf einen Sozialismus mit menschlichem Antlitz lief der Einschätzung Grušas zuwider. Seine nach 1989 geäußerte Hoffnung, dass nach der Samtenen Revolution von 1989 an die Ideen von 1968 angeknüpft werde, stand gleichfalls in einem Gegensatz zu Grušas Überzeugungen. Hier zeigt sich einmal mehr, dass gerade Memoiren, die ihren Fokus im Politischen setzen, nicht nur zu narratologischen, sondern auch zu inhaltlichen Konflikten führen. Thomas Couser hat darauf hingewiesen, dass die Verschränkung von zwei Sichten auf die Geschichte ethische Fragen aufwerfen und konflikthaft sein könne: „[There] is conflict between the writer's obligation to portray the subject as he or she would wish and the obligation to historical record." (Couser 2004, 45) In solchen Konflikten hat, wie im Fall Goldstücker, der Autobiografierte die Trümpfe in der Hand. In der tschechischen Ausgabe wird Gruša nur in einem Satz in seiner Eigenschaft als Schwiegersohn erwähnt. Der Konflikt zwischen Gruša und Goldstücker ist, um diesen Ausdruck Goldstückers zu gebrauchen, auch eine Spiegelung der tschechischen Wahrnehmung Goldstückers. Wie seine Töchter in ihrem Nachwort anmerken, stieß die Geschichte, die ihr Vater zu erzählen hatte, in den 1990er Jahren im Ausland auf mehr Interesse und Zustimmung als in Tschechien (Grušová und Vávrová 2005, 168).

Beide Versionen der Memoiren teilen trotz unterschiedlicher Leserausrichtung dieselbe Grundhaltung: Die Erinnerungen Goldstückers zeigen seine Loyalität den politischen Idealen seiner Jugend, aber auch der Partei gegenüber, an keiner Stelle sind sie entschuldigend oder defensiv. Er macht dies deutlich, wenn er den ersten Besuch seiner Frau im Gefängnis beschreibt:

Ich glaube niemand würde erraten, was nach achtzehn Monaten Trennung und angesichts der Aussicht auf einen lebenslänglichen Gefängnisaufenthalt die ersten Worte waren, die wir wechselten: „Unsere Einstellung zur Partei hat sich nicht geändert!“ (Goldstücker 1989, 227)

Und nach seiner Entlassung ist er - wie andere kommunistische Intellektuelle auch - überzeugt, dass die Erfahrung, die er während der Schauprozesse und im

17 Schreiber, der im Nachwort der Gespräche darauf hinweist, dass er nicht kommentiert oder korrigiert, ist im Hinblick auf die Bewertung des Sozialismus deutlich näher an den Überzeugungen Goldstückers, wie aus seinen Fragen erkennbar wird. 
Gefängnis gemacht hat, eine Folge des Stalinismus ist, der der tschechoslowakischen Kommunistischen Partei von der Sowjetunion aufoktroyiert wurde, und dass diese Fehlentwicklung das Projekt als Ganzes nicht diskreditiert. Die Beschreibung der Schauprozesse und des brutalen Antisemitismus der 1950er Jahre als „sowjetischer Import“ ist, wie Jiří Holý in einem Aufsatz zum Antizionismus der kommunistischen Ära herausarbeitet, ein gängiges tschechisches Narrativ (Holý 2016, 764), das den autochthonen Antisemitismus herunterspielt und verharmlost und sich zugleich eines Teils der Schuld entledigt. Goldstücker greift in ambivalenter Weise auf dieses Narrativ zurück, wenn er schreibt, die kommunistische Partei, seine Partei, in die er in den 1930er Jahren eingetreten sei, habe im Unterschied zu jener der 1950er und 1970er Jahre keinen Antisemitismus gekannt. Auch seine Beschreibung der Lagerhaft ist ambivalent: Die Jahre in Leopoldov und Jáchymov weisen zwar Motive der Lagerliteratur auf (Hunger, schlechtes Essen, Demütigungen, Kälte, Isolation), dennoch kann man sich aus den Erinnerungen Goldstückers kein wirkliches Bild davon machen, wie grausam und unmenschlich die Haftbedingungen in Jáchymov waren. Das hat zum einen etwas mit der schon erwähnten Grundhaltung des Textes zu tun, der sich nicht für das Individuelle, für Gefühle interessiert, sondern die Prozesse und die Haft im Kontext der großen Geschichte wahrnimmt. Zum anderen lässt sich diese Haltung jedoch auch als Verschweigen der dunklen Seiten des frühen tschechoslowakischen Nachkriegssozialismus lesen, welche weder zu Zweifeln noch zu einer Abkehr vom Marxismus oder der Partei führt. ${ }^{18}$ Dies ist eine Haltung, die Nanci Adler in ihrer Studie Keeping Faith with the Party. Communist Believers Return from the Gulag am Beispiel der Sowjetunion beschrieben und untersucht hat und die Eugen Ruge in seinem Roman In Zeiten abnehmenden Lichts (2011) thematisiert und die keineswegs selten anzutreffen ist. Noch in den 2000er Jahren wendet Goldstücker sich gegen die totale Abwertung der gesamten kommunistischen Ära und erklärt Eduard Schreiber:

Es gibt ein Gesetz, das deklariert, dass der Kommunismus eine verbrecherische Ideologie ist, und das bedeutet, dass ein jeder, der nicht rechtzeitig die Seiten änderte, grundsätzlich als Verbrecher anzusehen ist. Aus dem Kommunismus konnte also nichts Gutes kommen, auch wenn es die größten Werke der tschechischen Literatur in jener Zeit sind. (Goldstücker und Schreiber 2009, 71-72)

Goldstücker spielt hier auf das anti-kommunistische Narrativ an, das sich in der Bezeichnung der sozialistischen Ära als „totalita“ (Totalität) niederschlägt, das

18 Im Gespräch nach den Mitarbeitern der Staatssicherheit und ihren absurden Vorwürfen gefragt, sagt Goldstücker, man dachte, die sind Spezialisten, die müssen es wissen (Goldstücker und Gruša 1987/1988, Band 10). 
den Sozialismus als Fehlentwicklung und Ausreißer in der nationalen Geschichte darstellt und das von den kulturellen Eliten wie von der breiten Bevölkerung gepflegt wird (vgl. Valeš 2013). Anders als Gruša stellt Schreiber in seinem Text Goldstückers Loyalität der Partei gegenüber nicht in Frage und bewertet sie nicht. In der Interpretation von Wolfgang Müller-Funk und Jan Budňák ist dieses unerschütterliche Festhalten an seinen kommunistischen Überzeugungen ebenso „erstaunlich“ wie „mutig“ (Müller-Funk und Budňák 2013, 144), trägt aber aus ihrer Sicht letztlich dazu bei, die Erinnerungen zu entwerten. Sie können darin nichts „Positives“ sehen außer „Unerschrockenheit“ bei der Beschreibung seiner Rolle als Kommunist (Müller-Funk und Budňák 2013, 146). Ihrer Auffassung nach ist Gruša mit seinen Überlegungen zu Kommunismus und verblendetem Glauben näher an der Wahrheit als Goldstücker selbst (Müller-Funk und Budňák 2013, 153). In ihrer Wahrnehmung beeinträchtigt das anhaltende Bekenntnis zum Kommunismus seine Zeugenschaft (Müller-Funk und Budňák 2013, 146) und sie werfen ihm vor, keine Verantwortung für die Verbrechen des Kommunismus zu übernehmen. Sie lesen Goldstückers Parteikritik und seine Einschätzung, sein größter Fehler sei gewesen, die antisemitisch motivierte Zuweisung der Rolle des „falschen Zeugen“ (Goldstücker 1989, 343) akzeptiert zu haben, als Versuch sich freizusprechen von den Verbrechen des Kommunismus. Obwohl Goldstücker Fehleinschätzungen anspricht (Goldstücker 2004, 130-131), formuliert er kein dezidiertes Bekenntnis zur eigenen Schuld (weil es diese in seinen Augen nicht gibt). Der tschechische Text endet, wie bereits angemerkt, mit einer Auseinandersetzung mit dem Antisemitismus der Kommunistischen Partei der Tschechoslowakei, den Goldstücker jedoch keineswegs als zwangsläufige Folge, sondern als Fehlentwicklung darstellt. Im deutschen Text scheint dieser antisemitische Subtext noch in der Formulierung des „falschen Zeugen“ und damit in der Anspielung auf antisemitische Diskurse um die biblische Judasfigur durch. Ein Motiv, das Goldstücker in seinen Gesprächen mit Schreiber aufgreift und expliziert. Hier reflektiert er dies als „zurückgewiesene Loyalität“, als Loyalität zu einem „Objekt der Loyalität“, das „schon lange nicht mehr loyal ist“ (Goldstücker und Schreiber 2009, 142). Er bezeichnet diese „Bereitschaft zur Loyalität als ein Stück jüdischen Erbes“ (Goldstücker und Schreiber 2009, 142) und wendet sich damit gegen den antisemitischen Topos der Juden als aller - insbesondere nationaler - Bindungen ledig. Unter den verschiedenen identitären Elementen, die Goldstückers Biografie vereint, hebt er durch die prononcierte Positionierung dieser Passagen die jüdischen Anteile seiner Identität und diese wiederkehrenden Erfahrungen der „psychischen Enteignung“ (Goldstücker und Schreiber 2009, 143) hervor. Goldstücker weist darauf hin, wie schmerzlich für ihn der Einmarsch der Truppen des Warschauer Pakts und insbesondere der Sowjetunion gewesen sei. Aus seiner Perspektive war dies schlimmer als die Okkupation durch die deutschen Truppen im Krieg. 
Ich muss gestehen, dass ich diese Okkupation unvergleichlich viel härter empfand als die nationalsozialistische. Hitler war ein erklärter Feind [...]. Jetzt aber bebte der Boden unter den Stiefeln der Truppen des Staates, in den ich einst wie ins Gelobte Land gefahren war [...]. (Goldstücker 1989, 339) ${ }^{19}$

Der Einmarsch der Truppen der Warschauer Paktstaaten und die folgende antisemitische Kampagne führen zwar zu einer Desillusionierung, aber nicht zu einer vollständigen Abwendung von den sozialistischen Idealen, wie Goldstücker betont. Liest man seine Memoiren im Kontext der Erinnerungen anderer jüdischer Kommunisten in Mitteleuropa, so wird deutlich, dass seine Haltung ungewöhnlich ist. Für viele jüdische Intellektuelle stellen die antisemitischen Kampagnen des Jahres 1968 eine traumatische Wiederholung des Holocaust und den Auslöser für eine endgültige Abkehr vom Kommunismus dar.

\section{Mitteleuropäisch-jüdische Erinnerungen als transnationale Erinnerung}

$\mathrm{Zu}$ den Besonderheiten von Goldstückers Memoiren gehört es, dass sie trotz eines deutlichen Bekenntnisses zu einer tschechoslowakischen Identität nicht national geprägt sind. Goldstücker, der aus einer slowakisch-ungarisch-jüdischen Familie stammt, in Prag Romanistik und Germanistik studiert hat und zwei Mal in England im Exil war, versteht sich ganz im Sinne seiner kommunistischen Prägung in den 1930er Jahren als „Internationalist“. Dies manifestiert sich auch in den literarischen Referenzen, die er heranzieht: Sie reichen von Goethe bis Brecht, von Villon bis Gide, von Palacký bis Nezval. Goldstücker ist - für einen Literaturwissenschaftler - seltsam uninteressiert an den Spezifika der deutschen, englischen, israelischen Kultur, sie bleiben blass und treten hinter seinen Überlegungen zu gesellschaftlichen Verhältnissen und administrativen Vorgängen, aber auch parteiinternen Strategien zurück. Seine Erinnerungen sind nicht geprägt durch das, was Stefan Braese als „strukturelle Bipolarität“ (Braese 2014, 285) jüdischer Identitäten und Literaturen bezeichnet hat, die sich traditionell zwischen zwei Literaturen und Kulturen aufspannten, sondern durch eine Vielzahl von wechselnden Zuordnungen und Positionierungen. In der Erinnerung Goldstückers ist bereits die Familiengeschichte geprägt durch Transnationalität. Eine Transnationalität, die sich nicht nur durch Mobilität und Migration herstellt, wie im späteren Leben

19 „Invaze mě skutečně zaskočila, musím říct, že víc než nacistická okupace - nepřepadl nás zákeřný nepřitel, ale někdo, od koho jsme to nečekali.“ (Goldstücker 2005, 161) 
Goldstückers, sondern durch die Wechselfälle der Geschichte, die zu den jüdischen Prägungen noch ungarische und slowakische Einflüsse hinzutreten lässt. Einem ohnehin schon durch Transnationalität geprägten Leben fügen die beiden Exilerfahrungen noch weitere Dimensionen hinzu. Auffällig ist auch, dass Israel, dem zwar ein Kapitel der Erinnerungen gewidmet wird, danach als Referenz- und Bezugspunkt gänzlich aus dem Horizont Goldstückers verschwindet. Im Hinblick auf das tschechisch-deutsch-jüdische Prag vor 1938, das vielen als Inbegriff einer geglückten transnationalen Kultur gilt, äußert sich Goldstücker kritisch und merkt an, dies sei in manchen Hinsichten eine nachträgliche (nostalgische) Retusche (Goldstücker 2003, 87). ${ }^{20}$ Vielmehr drängen sich ihm immer wieder zahlreiche Bezüge zu einer europäischen Literatur und Kultur und einem Sozialismus auf, den er emphatisch als inter- und transnational aufgrund der Kooperationen über Sprach- und Staatsgrenzen hinweg versteht (Goldstücker 2003, 88).

Wie zu sehen war, stellt die Shoah dabei einen wichtigen, wenngleich nicht immer expliziten Bezugspunkt der literarisch reflektierten Erinnerungslandschaft Goldstückers dar. Es gibt jedoch in Goldstückers Erinnerungen keinen privilegierten Standort, von dem die Shoah in die geschichtliche Entwicklung integriert werden kann. Sie steht im Text scheinbar beziehungs- und ursachenlos. Goldstücker folgt mit seinen Erinnerungen den jüdischen Texten der literarischen Moderne, die, wie Gershon Shaked argumentiert hat, typischerweise zwischen einem (verweigerten) Assimilationsbegehren (Antisemitismus) und einer Tendenz zum Übernationalen und Universalistischen (Kommunismus), das jede konkrete Zugehörigkeit transzendiert, oszillieren (Shaked 1986, 204-205). Goldstückers Biografie ist insofern typisch für jüdische Intellektuelle in Mittelosteuropa, die häufig durch unterschiedliche Erfahrungswelten sowie mehrfache Loyalitäten und mehrfache nationale Zugehörigkeiten geprägt sind.

Diese Transnationalität wird in den verschiedenen Versionen seiner Memoiren noch verstärkt durch die nationalen Verortungen seiner Gesprächspartner, die sein Leben fragend rahmen und aus einer deutschen oder tschechischen Perspektive betrachten. Für Goldstücker, der für sich mehrfache Zugehörigkeiten in Anspruch nimmt, sind dies Vereinfachungen. Seine Bereitschaft, sich auf seine Gesprächspartner einzulassen, mit ihnen ins Gespräch zu kommen, ist aber auch ein Ausdruck einer grundlegend dialogischen Haltung. In seinen Memoiren

20 Manfred Weinberg hat dieser Wahrnehmung in seinem Text zu Goldstücker widersprochen. Seiner Meinung nach sind die „harten Grenzziehungen [...] - etwa was die völlige Gesprächslosigkeit der Deutschen mit den Tschechen, aber auch die strikte Gegenüberstellung von Prager deutscher und sudetendeutscher Literatur angeht - durch gründliche, zunächst historische Forschungen inzwischen obsolet“ (Weinberg 2013, 119). 
treffen verschiedene Narrative aufeinander und schaffen eine Konstellation, die ebenso transnational ist wie seine mittelosteuropäisch-jüdische Identität. Goldstückers Erinnerungen sind transversal, sie verbinden Räume, Kontexte und Kulturen (Rothberg 2009, 18) und erweisen sich so in einem affektiven Sinn als nicht mehr national, wohl aber historisch durch die Zeitläufte gebunden. Goldstücker ist in seiner Eigenschaft als Literaturhistoriker immer darum bemüht, Ereignisse einzuordnen und in einen historischen Rahmen zu stellen, der das Individuelle hinter das Allgemeine zurücktreten lässt. Diese Haltung kommt im Epilog der deutschen Ausgabe nochmals deutlich zum Ausdruck: „Du, der du dir einbildest, dass du ein unabhängiges, individuelles Leben gestaltest, bist am Ende nur die Verkörperung eines Schicksalstypus. Eines mitteleuropäischen Intellektuellen aus dem entwurzelten Mitteleuropa.“ (Goldstücker 1989, 343) Goldstücker ist bis an sein Lebensende der festen Überzeugung, dass die Spezifik im Universalen aufhebbar ist und dass es sich in der entleerten Abstraktion des Übernationalen (gut) leben lässt.

\section{Literaturverzeichnis}

\section{Primärliteratur}

Goldstücker, Eduard. Prozesse. Erfahrungen eines Mitteleuropäers. Aus dem Tschechischen übersetzt von Friedrich Uttitz. München, Hamburg: Knaus, 1989.

Goldstücker, Eduard. Vzpomínky (1913-1945). Praha: G plus G, 2003.

Goldstücker, Eduard. Vzpomínky (1945-1968). Praha: G plus G, 2005.

Goldstücker, Eduard, und Jiři Gruša. Tonbandaufnahmen mit biografischen Interviews. Unveröffentlicht. 1987/1988. Bestand des literarischen Nachlassarchivs des Mährischen Landesmuseums Brno, Nachlassarchiv von Jiři Gruša.

Goldstücker, Eduard, und Eduard Schreiber. Von der Stunde der Hoffnung zur Stunde des Nichts. Gespräche. Wuppertal: Arco, 2009.

Gruša, Jiří, und Dalibor Dobiáš. Umění stárnout. Praha, Litomyšl: Paseka, 2004. Grušová, Anna, und Helena Vávrová. „Po roce 1968 (pohledem dcer)“. Eduard Goldstücker. Vzpomínky (1945-1968). Praha: G plus G, 2005. 165-171.

\section{Sekundärliteratur}

Adler, Nanci. Keeping Faith with the Party. Communist Believers Return from the Gulag. Bloomington: Indiana University Press, 2012.

Adler, Nanci. „Enduring Repression: Narratives of Loyalty to the Party before, during and after the Gulag“. Europa-Asia Studies 62.2 (2010): 211-234. 
Billson, Marcus. „Memoiren. Neue Perspektiven auf eine vernachlässigte Gattung“. Texte zur Theorie der Auto/Biographie. Hgg. Anja Tippner, Christopher F. Laferl. Stuttgart: Reclam Verlag, 2016. 138-163.

Braese, Stephan. „Blutet er nicht? Shylock als Faszinosum jüdischer Künstler“. Yearbook for European Jewish Literature Studies 1 (2014): 280-294.

Couser, Thomas. Vulnerable Subjects. Ethics and Life Writing. Ithaca, London: Cornell University Press, 2004.

Genette, Gérard. Das Buch vom Beiwerk des Buches. Frankfurt am Main, New York: Campus, 1989.

Gilman, Sander. „Gibt es neue Ostjuden in der deutsch-jüdischen Gegenwartsliteratur?“. Konzept Osteuropa: Der „Osten“ als Konstrukt der Fremd- und Eigenbestimmung in deutschsprachigen Texten des 19. und 20. Jahrhunderts. Hgg. Dagmar Lorenz, Ingrid Spörk. Würzburg: Königshausen \& Neumann, 2011. 259-279.

Holý, jiří. „,Antisionismus“ jako skrytý a zjevný antisemitismus doby komunistického režimu“. Cizí i blizcí: Židé, literatura, kultura v českých zemích ve 20. století. Hg. Jiří Holý. Praha: Akropolis, 2016. 761-797.

Hudabiunigg, Ingrid. „Eduard Goldstückers Hoffnung auf einen ,Sozialismus mit menschlichem Antlitz“". Exile in and from Czechoslovakia during the 1930s and 1940s. Hgg. Charmian Brinson, Marian Malet. Amsterdam: Rodopi, 2010. 267-288.

Müller-Funk, Wolfgang, und Jan Budňák. „Die zweite Spiegelung. Jiři Gruša als der Andere in Eduard Goldstückers Prozesse. Erfahrungen eines Mitteleuropäers / Vzpomínky.“ brücken: Germanistisches Jahrbuch Tschechien-Slowakei 21.1-2 (2013): 140-155.

Niethammer, Lutz. „Fragen - Antworten - Fragen. Methodische Erfahrungen und Erwägungen zur Oral History“. Oral History. Basistexte. Hg. Julia Obertreis. Stuttgart: Franz Steiner Verlag, 2012. 31-73.

Rothberg, Michael. Multidirectional Memory: Remembering the Holocaust in the Age of Decolonization. Stanford: Stanford University Press, 2009.

Shaked, Gershon. Die Macht der Identität: Essays über jüdische Schriftsteller. Königstein/Ts.: Jüdischer Verlag bei Athenäum, 1986.

Stone, Albert E. „Collaboration in Contemporary American Autobiography“. Revue française d'études américaines 14 (1982): 151-165.

Tippner, Anja. „Transnationalität und Erinnerung“. Kulturwissenschaftliches Handbuch Literatur und Transnationalität. Hgg. Doerte Bischoff, Susanne Komfort-Hein. Berlin: De Gruyter, 2019. 153-167.

Tuckerová, Veronika. „Reading Kafka, Writing Vita: The Trials of the Kafka Scholar Eduard Goldstücker". New German Critique 42.1 (2015): 129-161.

Valeš, Lukáš. „Antikomunismus jako nová politická ideologie“. Společenskovědní aspekty fenoménu vyrovnání se minulostí v kontextu výchovy k občanství. Hg. Pavel Kopeček et al. Praha: Nakladatelství Epocha, 2013. 60-81.

Weinberg, Manfred. „Eduard Goldstücker: Das wiederaufgenommene Gespräch. Blicke auf einen Europäer des 20. Jahrhunderts. Einleitung“. brücken. Germanistisches Jahrbuch Tschechien-Slowakei 21.1-2 (2013): 114-124.

Wirth, Uwe. „Das Vorwort als performative, paratextuelle und parergonale Rahmung“. Rhetorik. Figuration und Performanz. Hg. Jürgen Fohrmann. Stuttgart, Weimar: Metzler Verlag, 2004. 603-628. 